\title{
Back pain in the older patient
}

\author{
E Baumgartner, L Heitmann, B Duvoisin, A K L So
}

^Department of

Diagnostic Imaging, The

Robert Jones and Agnes

Hunt Orthopaedic

Hospital, Oswestry,

Shropshire SY10 7AG,

United Kingdom.

\section{Service de}

Rhumatologie, Centre

Hospitalier

Universitaire Vaudois,

Lausanne, Switzerland

E Baumgartner

L Heitmann

A K L So

Service de Radiologie, Centre Hospitalier

Universitaire Vaudois

Lausanne, Switzerland

B Duvoisin

Correspondence to:

Professor A K L So, Service de Rhumatologie, CHUV,

CH-1011 Lausanne,

Switzerland.

Accepted for publication 9 January 1996

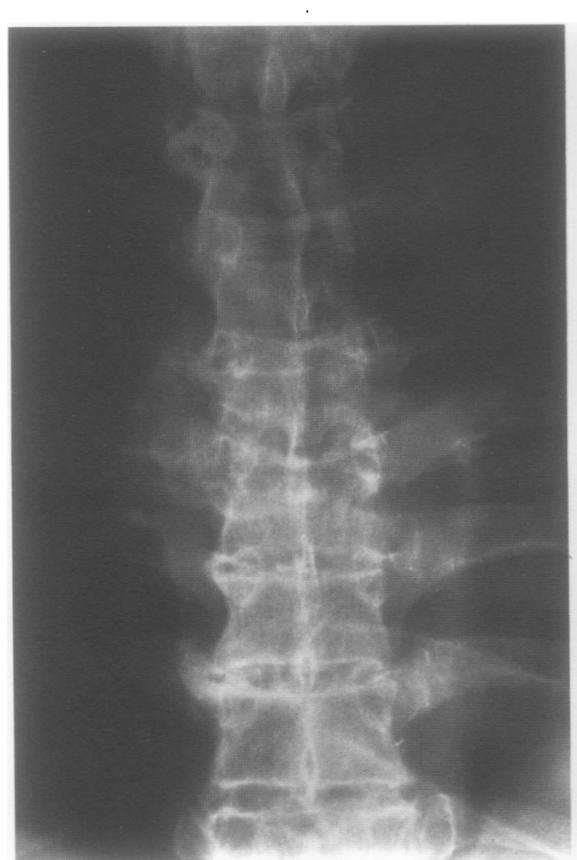

\section{Clinical history}

A 72 year old male patient presented with recent onset thoracic back pain. The pain was continuous and intense, exacerbated by movements of the spine, and radiated bilaterally to the costal region. The patient was known to suffer from non-insulin-dependent diabetes mellitus and hypertension. On admission, he appeared unwell and had a fever of $37.5^{\circ} \mathrm{C}$ Clinical examination revealed localised tenderness at the $\mathrm{T} 7$ level, with bilateral radicular dysaesthesia in the T7-8 area.

Conventional radiographs of the thoracic spine were interpreted as showing diffuse idiopathic skeletal hyperostosis (DISH) and degenerative spondylosis (fig 1). The patient was treated with non-steroidal antiinflammatory drugs, acetaminophen, and muscle relaxants, with improvement of pain, and was discharged from the original admitting hospital three weeks later.

Seven days after discharge, he was referred to our hospital because of an acute relapse of thoracic pain associated with febrile episodes. On examination, there was severe tenderness over T6-8, with hypoaesthesia in the T6-7 distribution bilaterally. There were no signs of

Figure 1 Conventional thoracic spine radiographs. Left: Frontal view. The T7-8 disc is narrowed, with irregular, ill defined endplates. Paravertebral soft tissue swelling is evident, suggesting paravertebral abscess. Note also spondylosis. Right: Left lateral view. The T7-8 disc is indistinct. Radiological changes of diffuse idiopathic skeletal hyperostosis are evident. spinal cord compression. The temperature was normal on his entry to hospital. The leucocyte count was $10.1 \mathrm{~g} \mathrm{l}^{-1}$, erythrocyte sedimentation rate (ESR) $125 \mathrm{~mm}$ in the first hour, and $\mathrm{C}$ reactive protein $140 \mathrm{mg} \mathrm{l}^{-1}$ (normal range $0-10$ $\left.\mathrm{mg} \mathrm{l}^{-1}\right)$.

\section{Radiological findings and diagnosis}

Plain radiographs were performed and a magnetic resonance imaging (MRI) scan was requested.

On frontal view, the plain film showed a narrowed T7-8 disc space, with irregular and ill defined endplates. Paravertebral soft tissue swelling was evident. The lateral view showed an indistinct T7-8 intervertebral disc with radiological changes characteristic of DISH (fig 1).

The MRI performed on the day of admission revealed vertebral changes suggestive of T7-8 infective spondylodiscitis: an abnormal signal suggesting oedema and fibrovascular tissue was observed in both vertebral bodies, with a narrowed disc space. Inflammatory soft tissue swelling was demonstrated in the paravertebral and epidural space of the spinal canal, with abscess formation (fig 2).

Streptococcus bovis was identified on blood cultures and confirmed by needle biopsy of the intervertebral disc. Treatment with intravenous penicillin and netilmycin was started; the pain disappeared completely within one week, and this was accompanied by neurological recovery. Intravenous penicillin was continued for six weeks, then substituted by an oral regimen of amoxycillin for a further six weeks. At 10 week follow up, the patient was well, and the ESR and leucocyte count had normalised. Plain radiographs were performed six months after the treatment ended, and showed a slight progression of the narrowing of the T7-8 intervertebral space, with the endplates being more irregular and condensed (fig 3). There was no vertebral fusion or important deformity.

\section{Discussion}

Spinal epidural abscess is a rare cause of back pain; its estimated prevalence in primary care patients with acute low back pain is $0.004 \%{ }^{1}$ Early treatment is essential as, untreated, this condition can lead to paralysis and death.

Unfortunately, the diagnosis of spinal epidural abscess is often delayed, with fewer 
than $40 \%$ of cases being diagnosed at the time of the patient's admission to hospital..$^{2-5}$ Back pain is invariably present; other symptoms and physical signs are varied and include fever, weakness, radicular pain, sensory loss, paralysis, sphincter dysfunction, stiffness, and encephalopathy. ${ }^{23}$

Our patient's clinical presentation with acute onset of severe and continuous pain, general malaise with fever, and well localised vertebral tenderness, pointed to an infectious process until proved otherwise.

As described above, conventional radiographs of the thoracic spine showed changes compatible with DISH (fig 1). This condition is a frequent radiological finding in the elderly, especially if there is a history of diabetes mellitus (present in $40 \%$ of patients with DISH). Conversely, DISH is found in approximatively $20 \%$ of patients with diabetes. ${ }^{6}$ According to a recent study, there is no significant difference in the frequency of back pain at any spinal level between patients with DISH and control subjects. ${ }^{7}$ However, it is well known that spinal DISH may occasionally lead to stenosis of the spinal canal.

A known history of diabetes mellitus is noteworthy in the case of our patient. Associated factors can be found in up to $80 \%$ of patients with spinal epidural abscess, and diabetes is the most frequent underlying disease encountered, often in cases with multifocal involvement. ${ }^{2-4} 8$ Other predisposing conditions include intravenous drug abuse, alcoholism, obesity, and immunosuppression.

The time from onset of symptoms to hospital admission varies widely (range 1 to 60 days), ${ }^{2}$ to the point that some authors claim to differentiate between acute and chronic forms. ${ }^{3}$ Even in insidious, slowly progressing spinal epidural abscess, a rapid catastrophic evolution with paralysis or septic shock may occur at any time.

In our patient, the causative organism was Streptococcus bovis. This organism is a very rare aetiological agent of spinal epidural abscess, but other streptococcal species account for up to $20 \%$ of the infective organisms. ${ }^{5-8}$ The port of entry into the bloodstream in our patient remains unknown, as extensive investigations, including a colonoscopy, were normal.

Although clinical diagnosis can be aided by radiological demonstration of the abscess and associated bony and soft tissue changes, spinal epidural abscess still represents a diagnostic challenge, even in the era of computed tomography (CT) and MRI. Conventional radiographs of the spine show abnormalities in only $44-65 \%$ of the patients, in the form of osteomyelitis and paravertebral soft tissue
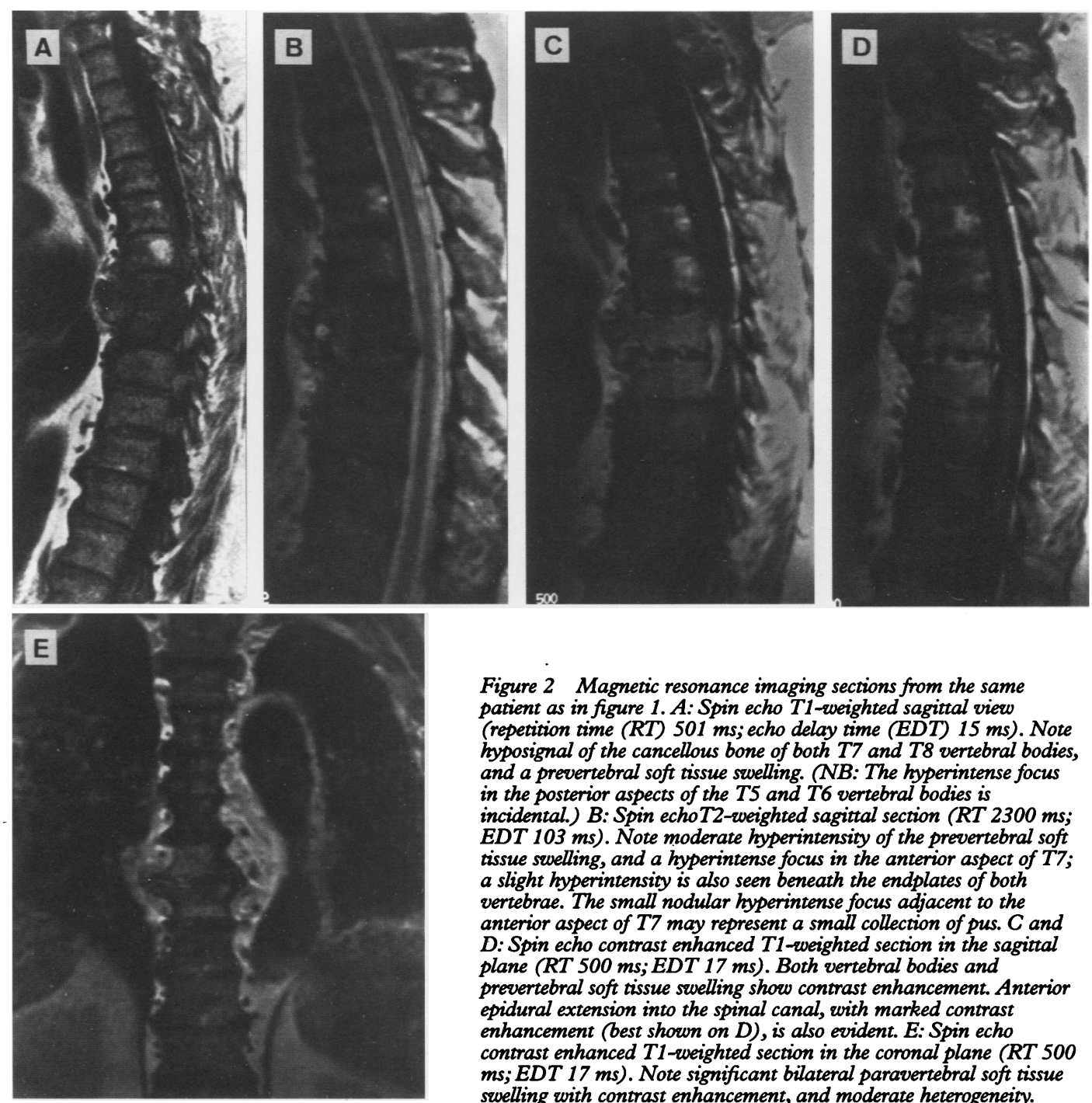

Figure 2 Magnetic resonance imaging sections from the same patient as in figure 1. A: Spin echo T1-weighted sagittal view (repetition time (RT) $501 \mathrm{~ms}$; echo delay time (EDT) $15 \mathrm{~ms}$ ). Note hyposignal of the cancellous bone of both $T 7$ and $T 8$ vertebral bodies, and a prevertebral soft tissue swelling. (NB: The hyperintense focus in the posterior aspects of the T5 and T6 vertebral bodies is incidental.) B: Spin echoT2-weighted sagittal section (RT $2300 \mathrm{~ms}$; EDT $103 \mathrm{~ms}$ ). Note moderate hyperintensity of the prevertebral soft tissue swelling, and a hyperintense focus in the anterior aspect of T7; a slight hyperintensity is also seen beneath the endplates of both vertebrae. The small nodular hyperintense focus adjacent to the anterior aspect of $T 7$ may represent a small collection of pus. $C$ and $D:$ Spin echo contrast enhanced T1-weighted section in the sagittal plane (RT $500 \mathrm{~ms} ; E D T 17 \mathrm{~ms}$ ). Both vertebral bodies and prevertebral soft tissue swelling show contrast enhancement. Anterior epidural extension into the spinal canal, with marked contrast enhancement (best shown on D), is also evident. E: Spin echo contrast enhanced T1-weighted section in the coronal plane (RT 500 ms; EDT $17 \mathrm{~ms}$ ). Note significant bilateral paravertebral soft tissue swelling with contrast enhancement, and moderate heterogeneity. 
oedema. ${ }^{2-3}$ Positive radiological studies have increased considerably with techniques such as CT myelography and, more recently, MRI. These radiological investigations allow positive findings in $80-100 \%$ of the patients. ${ }^{2-5}$ When available, MRI presents several advantages in comparison with CT myelography: for example, the technique is not invasive, while inadvertent puncture of a spinal epidural abscess during preparation for myelography could lead to seeding into the cerebrospinal fluid and iatrogenic bacterial meningitis. ${ }^{9} \mathrm{MRI}$ provides an optimal analysis of various tissues, including the spinal cord, the intervertebral discs, the vertebrae, and the adjacent soft tissues. Contrast enhanced MRI with gadolinium allows recognition of pus and granulation tissue, thus giving an accurate idea of the topographic localisation and extent of the spinal epidural abscess, leading to better differentiation of the abscess from the compressed or infected subarachnoid space. Moreover, detection of gadolinium uptake in the involved disc, vertebrae, or both, facilitates the diagnosis of concomitant spondylodiscitis or osteomyelitis. ${ }^{10} \mathrm{MRI}$ is also well suited for follow up studies to demonstrate any decrease or increase in size of the abscess, especially when the treatment is non-surgical. The sensitivity of MRI for spinal epidural abscess is comparable to that of CT myelography, ${ }^{3}$ though no definitive data are available concerning specificity, which should be superior to that of CT myelography. ${ }^{11}$ The latter technique should be performed in emergency situations, especially if MRI is not readily available. In the presence of concomitant bacterial meningitis, MRI is probably inferior to CT myelography, as the subarachnoid and epidural spaces can be differentiated only with difficulty. ${ }^{-12}$

Radionuclide bone scans, with technetium$99 \mathrm{~m}$ diphosphonates, gallium, or labelled leucocytes, can also be used to diagnose spinal epidural abscess, but were not performed in
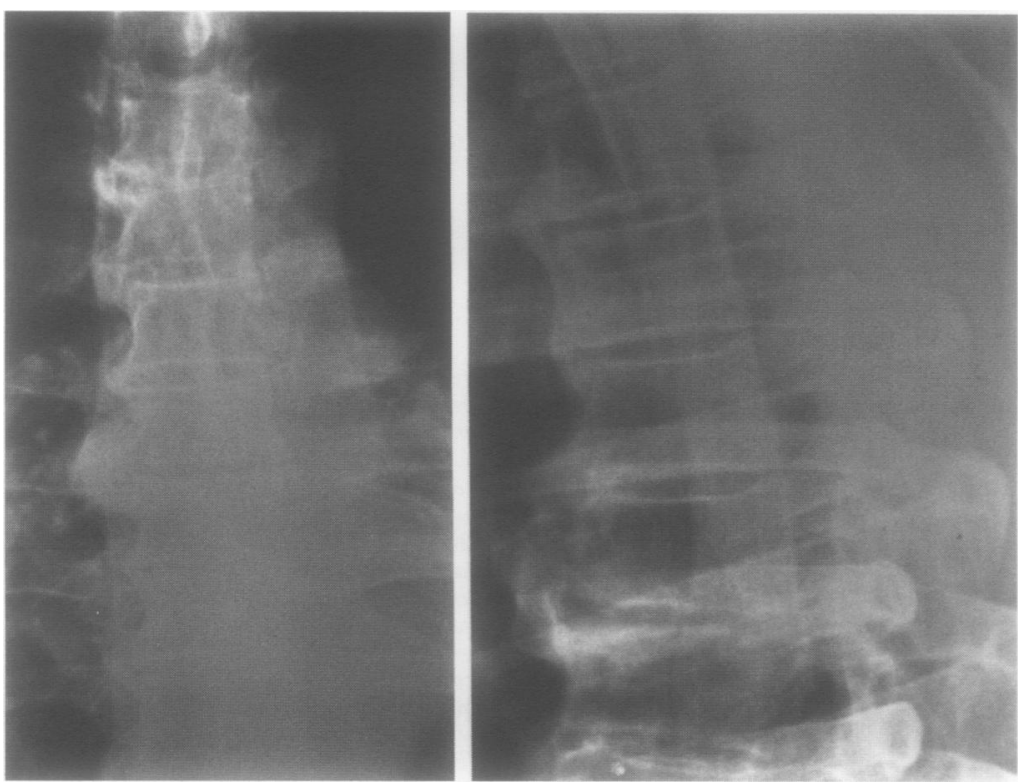

Figure 3 Conventional thoracic spine radiographs six months after treatment. Left: Frontal view. The narrowing of the $T 7-8$ disc is slightly more pronounced and the lateral hyperostosis seems more prominent. Right: Right lateral view. The endplates are more irregular and sclerosis has increased. our patient. Recent studies have shown no advantage of these techniques over CT myelography and MRI, as they provide poor information on the soft tissue extent of the infective process and its effect on neurological structures. $^{2-3}$ Scintigraphy, however, is very sensitive, though with a low specificity: it is particularly useful in demonstrating multifocal spinal involvement, but lacks the structural detail provided by MRI.

Surgical treatment has been the standard approach for spinal epidural abscesses. Despite improvement in diagnostic procedures, the rate of death and paralysis is still high, and reached $20-25 \%$ in two recent series. ${ }^{2-3}$ Paralysis is usually irreversible, especially if surgery is delayed for more than 24 hours. Recent reports, ${ }^{13-14}$ including a meta-analysis, ${ }^{15}$ have shown that non-surgical management can be successful in a subpopulation of patients with no or incomplete neurological deficit. Despite the lack of prospective, randomised trials comparing surgical and non-surgical treatments for this group of patients, it is likely that nonoperative management constitutes a good alternative to surgery for selected patients.

Prolonged courses of parenteral antibiotics and subsequent oral administration are needed to avoid relapse of the infection. However, there are no firm data as to the required duration of treatment.

The patient described illustrates the value of MRI in the diagnosis of this condition, and the successful outcome with early conservative treatment.

1 Deyo R A. Early diagnostic evaluation of low back pain. $f$ Gen Int Med 1986; 1: 328-38.

2 Darouiche R O, Hamill R J, Greenberg S B, Weathers S W, Musher D M. Bacterial spinal epidural abscess. Review of 43 cases and literature survey. Medicine 1992; 71: 369-85.

3 Hlavin M L, Kaminski H J, Ross J S, Ganz E. Spinal epidural abscess: a ten-year erspective. Neurosurgery 1990 27: 177-84.

4 Nussbaum E S, Rigamonti D, Standiford H, Numaguchi Y, Wolf A L, Robinson W L. Spinal epidural abscess: a report of 40 cases and review. Surg Neurol 1992; 38: 225-31.

5 Maslen D R, Jones S R, Crislip M A, Bracis R, Dworkin R J, Flemming J E. Spinal dural abscess. Optimising patient care. Arch Intern Med 1993; 153: 1713-21.

6 Mazières B, Arlet J, Belleville A. Hyperostose vertébrale, obésité et diabète. Rhumatologie 1981; 3: 135-7.

7 Schlapbach P, Beyeler C H, Gerber N J, et al. Diffuse idiopathic skeletal hyperostosis (DISH) of the spine: a cause of back pain? A controlled study. $\mathrm{Br} \mathcal{f}$ Rheumato 1989; 28: 299-303.

8 Del Curling O Jr, Gower D J, McWhorter J M. Changing concepts in spinal epidural abscess: a report of 29 cases. Neurosurgery 1990; 27: 185-92.

9 Teman A J. Spinal epidural abscess. Early detection with gadolinium magnetic resonance imaging. Arch Neurol gadolinium mag

10 Sadato N, Numaguchi Y, Rigamonti D, et al. Spinal epidural abscess with gadolinium-enhanced MRI: serial follow-up studies and clinical correlations. Neuroradiology 1994; 36: 44-8.

11 Erntell M, Holtas S, Norlin K, Dahlquist E, Nilsson-Ehle I Magnetic imaging in the diagnosis of spinal epidura abscess. Scand $\mathcal{F}$ Infect Dis 1988; 20: 323-7.

12 Danner $R$ L, Hartmann B J. Update of spinal epidural abscess: 35 cases and review of the literature. Rev Infect Dis 1987; 9: 265-74.

13 Mampalam T J, Rosegay H, Andrews B T, Rosenblum M L Pitts $L$ H. Nonoperative treatment of spinal epidural Pitts L H. Nonoperative treatment of

14 Leys D, Lesoin F, Viaud C, et al. Decreased morbidity from acute bacterial spinal epidural abscesses using computed tomography and nonsurgical treatment in selected patients. Ann Neurol 1985; 17: 350-5.

15 Wheeler D, Keiser P, Rigamonti D, Keay S. Medica management of spinal epidural abscesses: case report and review. Clin Infect Dis 1992; 15: 22-7. 